\title{
Glomerular Filtration Rate and Error Calculation Based on the Slope-Intercept Method with Chromium-51 Ethylenediaminetetraacetic Acid via a New Clinical Software: GFRcalc
}

\author{
Barbara Katharina Geist ${ }^{\mathrm{a}}$ Markus Diemling ${ }^{\mathrm{b}}$ Anton Staudenherz ${ }^{\mathrm{a}}$ \\ a Department of Biomedical Imaging and Image-Guided Therapy, Medical University of Vienna, Vienna, Austria; \\ ${ }^{b}$ Hermes Medical Solutions, Stockholm, Sweden
}

\section{Key Words}

${ }^{51} \mathrm{Cr}$-EDTA · Error calculation · Glomerular filtration rate .

Slope-intercept method · Software

\begin{abstract}
Objective: To evaluate a home-built Java-based program (GFRcalc) to simplify the calculation of glomerular filtration rate (GFR) after administration of chromium-51 ethylenediaminetetraacetic acid $\left({ }^{51} \mathrm{Cr}\right.$-EDTA) for routine clinical use. Materials and Methods: In the program GFRcalc, the GFR was calculated based on the biological half-life of the ${ }^{51} \mathrm{Cr}$ EDTA concentration using the slope-intercept method of between two and five blood samples. Additional features included the ability to export patient data and generate clinical reports as well as to calculate the error of the fit of the GFR measurement in cases with three or more blood samples collected. The GFR was calculated from one, two and three blood samples of 133 patients with body surface-corrected GFR of $21-213 \mathrm{ml} / \mathrm{min} / 1.73 \mathrm{~m}^{2}$. The Pearson correlation coefficient and the error of the fit for the GFR measurement were calculated for the three-sample method. Results: The correlation coefficient for the three-sample method and the fit error correlated well for small fit errors; in case of fit errors $>10 \%$, the correlation coefficient partially differed in results
\end{abstract}

compared to the other methods. The three-sample GFR values differed by approximately $17 \%$ from the single-sample GFRs. The fit errors of the three-sample GFRs correlated ( $r=$ 0.57) with their difference from the two-sample GFRs. Conclusion: In this study, the fit error that GFRcalc provided for the three-sample GFR offered a simple and reliable method to check the results obtained. This could also allow physicians to assess the reliability of the results and base their decisions on the quality of the measurement.

(C) 2016 S. Karger AG, Basel

\section{Introduction}

Since the late 1960s, chromium-51 ethylenediaminetetraacetic acid $\left({ }^{51} \mathrm{Cr}\right.$-EDTA) clearance measurements with two or more blood samples have been used to obtain an estimation of glomerular filtration rate (GFR) of the human kidney $[1,2]$. However, its routine use was hampered by the complexity and the effort required to calculate it. Useful routine indications include [3]: calculation of the maximum dose for chemotherapy based on the function of the kidney particularly in pediatric oncology, monitoring kidney function in nephrotoxic drug therapies, detection of renal failure prior to increases in serum

\begin{tabular}{ll}
\hline KARGER & $\begin{array}{l}\text { ○ 2016 S. Karger AG, Basel } \\
1011-7571 / 16 / 0254-0368 \$ 39.50 / 0 \quad \text { Karger }\end{array}$ \\
E-Mail karger@karger.com & $\begin{array}{l}\text { Thisis an Open Access article licensed under the terms of the } \\
\text { Creative Commons Attribution-NonCommercial 3.0 Un- } \\
\text { ported license (CC BY-NC) (www.karger.com/OA-license), } \\
\text { applicable to the online version of the article only. Distribu- } \\
\text { tion permitted for non-commercial purposes only. }\end{array}$
\end{tabular}

Barbara Katharina Geist

Department of Biomedical Imaging and Image-Guided Therapy

Medical University of Vienna, Währinger Gürtel 18-20

AT-1090 Vienna (Austria)

E-Mail barbara.geist@meduniwien.ac.at 
creatinine, assessment of potential live donors for kidney transplantation and evaluation of single kidney function in conjunction with relative renal function measurements using static or dynamic radionuclide imaging.

The GFR measurement determined from plasma ${ }^{51} \mathrm{Cr}$ EDTA concentrations is a reliable method for these needs [4]. The slope-intercept method, which is based on at least two blood samples, is routinely used in many hospitals worldwide [4]. It may be helpful to assess the quality of the GFR calculations and measurements, since systematic errors, such as those arising from the clinical procedure or from the measurement devices, may be encountered. In particular when statistical (i.e. random) errors become negligible, irregularities in the measurement process can be detected by a reliable error calculation. Therefore, the objective of this study was to evaluate a homebuilt Java-based software program (GFRcalc) that included a calculation of the error of the fit for three blood samples.

\section{Materials and Methods}

The program GFRcalc was separated into four subprograms, of which each appeared in its own tab folder: GFR calculation, GFR comparison, PDF viewer and settings. The first program was used for this study; the other programs, in principle, allowed the comparison of patient results, as well as to view, print and modify a clinical report, which was generated for each patient. The software was originally developed for use in the Division of Nuclear Medicine, Department of Biomedical Imaging and Image-Guided Therapy, Vienna General Hospital. It was written in Java, which allows a platform-independent performance.

\section{GFR Calculation}

The first subprogram calculated the absolute and the body surface area (BSA)-corrected GFR from the plasma concentration of ${ }^{51} \mathrm{Cr}$-EDTA in the blood samples of 133 patients using the slopeintercept method. Following intravenous administration of ${ }^{51} \mathrm{Cr}$ EDTA, three blood samples were taken from each patient. The collection times were 120,180 and 240 min after injection. For the calculation of the GFR based on two blood samples, the samples were taken after 180 and $240 \mathrm{~min}$ from each patient. For the determination of ${ }^{51} \mathrm{Cr}$-EDTA clearance in the blood, the decay of ${ }^{51} \mathrm{Cr}$ EDTA was measured based on the radioactivity of the Cr-isotope in the blood and evaluated with the slope-intercept method [4]. To overcome the initial distribution in the body, the first blood sample was always collected $120 \mathrm{~min}$ following ${ }^{51} \mathrm{Cr}$-EDTA administration. Additionally, several corrections were made in the program for the missing area under the plasma concentration versus time curve associated with the initial exponential, which was present when multiple blood samples were collected from earlier time points. The correction formulae included the formulae of Brochner-Mortensen for adults and children $[5,6]$, and also two modified versions of the formulae $[4,7]$. Besides biological processes, the count loss of ${ }^{51} \mathrm{Cr}$ due to radioactive decay was taken as negli- gible due to its long physical half-life of 27.7 days, resulting in a total count loss of $1 \%$ during the whole examination procedure. Consequently, this isotope was treated as a radiation source with a constant decay rate and the biological half-life of ${ }^{51} \mathrm{Cr}$-EDTA was determined with a mono-exponential fit through the data points of its concentrations in the blood samples.

After entering patient-relevant data (name, height, age and weight) into the program, details of the blood samples were specified: (a) the number of blood samples from which GFR was calculated, (b) the exact times of sample collection, (c) the blood sample volume and the standard dilution, $(\mathrm{d})$ the reading (in counts per minute, cpm) of the full syringe measurement (performed on a gamma camera or a gamma probe), including background and standard, and (e) the cpm reading of the blood samples (performed in a well counter), including background and standard. The program allowed one to three single measurements for each per blood sample; the mean value was used in the algorithm. Since GFR varies with BSA [4], the BSA-corrected GFR (BSA-GFR) normalized to the 'standard human' (BSA $1.73 \mathrm{~m}^{2}$ ) was also calculated. Several correction formulae have been published: Mosteller [8] (which was taken for the present calculations), DuBois and DuBois [9], Haycock et al. [10], Gehan and George [11] or Boyd [12]. If the number of blood samples collected was three or higher, an error of the fit calculation was provided. This limitation arises from the fact that in the case of two samples, the number of fit parameters was equal to the number of data points, which always leads to a unique solution of the exponential curve. A typical screen shot of the program presenting the plot of the mono-exponential fit, the calculated GFR, the BSA-GFR and the error of the fit is displayed in figure 1. The results and corresponding input data were stored in a database, which was also used to transfer existing data into the program in order to repeat a calculation or check previous results. In addition, a medical report specified for the attending physician, which contains the patient data, the results of the GFR calculation and the plot of the exponential fit, was generated for each selected patient.

\section{Mathematical Background}

GFR was calculated as the total dose of ${ }^{51} \mathrm{Cr}$-EDTA administered to the patient divided by the area under the plasma concentration versus time curve $[\mathrm{P}(\mathrm{t})]$. The curve was obtained using the slope-intercept method as specified in the British Nuclear Medicine Society guidelines [4]. The following formula was used to calculate the uncorrected GFR:

$$
G F R=-D \cdot \frac{C_{S t d}}{S_{S t d}} \cdot V \cdot \frac{\lambda}{P_{0}},
$$

where $\lambda$ is the biological mean lifetime and $\mathrm{P}_{0}$ the initial plasma concentration; both values were obtained by the fit procedure (see equation 2). $\mathrm{D}$ is the difference between the measurements of the full and the empty syringe, $\mathrm{C}_{\mathrm{Std}}$ is the cpm measurement of the standard, $\mathrm{S}_{\mathrm{Std}}$ is the probe head or gamma camera measurement of the standard and $\mathrm{V}$ the dilution of the blood sample (i.e. 500).

All quantities measured in the counter or the gamma camera were corrected for background.

\section{Calculation of the Fit of the Line of the Slope}

From the blood samples, a set of $n(n=2, \ldots, 5)$ data points at the times $t_{i}(i=1, \ldots, n)$ was obtained, each with a measured plasma concentration of $\mathrm{P}_{\mathrm{i}}$. Since the count rate of the blood samples mea- 


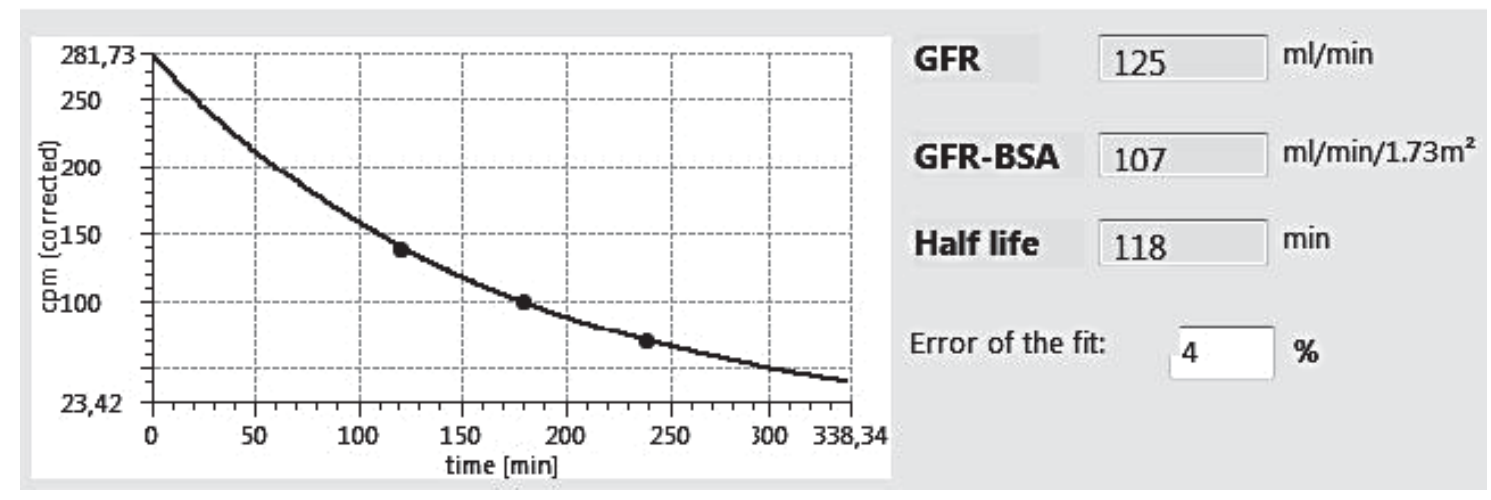

Fig. 1. Screen shot of part of the first subprogram GFRcalculation presenting the results of the calculation. The graph shows the cpm of ${ }^{51} \mathrm{Cr}$-EDTA in the blood samples (dots) and the exponential fit curve (line).

sured decreases approximately mono-exponentially, the plasma concentration curve appears as:

$$
P(t)=P_{0} \cdot e^{-\lambda t}
$$

where $\mathrm{P}(\mathrm{t})$ is the plasma concentration at a certain time $\mathrm{t}, \mathrm{P}_{0}$ represents the initial plasma concentration and $\lambda$ the biological mean lifetime defined as $\ln (2)$ divided by the biological half-life.

The exponential fit - and therefore the parameters $\mathrm{P}_{0}$ and $\lambda-$ was then obtained via the method of least squares where the optimum curve through a set of given data points is calculated.

\section{Calculation of the Fit Error}

The calculated error was the uncertainty of the fit of the line through the data points and was determined from the variance of the measured data points from the calculated curve. For this purpose, the area under the curve needs to be calculated as:

$$
A=\frac{P_{0}}{\lambda},
$$

where $\mathrm{A}$ is the area under the curve, $\mathrm{P}_{0}$ the calculated initial number of counts and $\lambda$ the mean lifetime. According to the Gaussian error propagation law, the errors of $\mathrm{P}_{0}\left(\sigma_{\mathrm{P}_{0}}\right)$ and $\lambda\left(\sigma_{\lambda}\right)$ must be calculated in order to get the error of the area under the curve $\sigma_{\mathrm{A}}$ :

$$
\sigma_{\alpha}=\sqrt{\left(\sigma_{P_{0}} \frac{1}{\lambda}\right)^{2}+\left(\sigma_{\lambda} \frac{P_{0}}{\lambda^{2}}\right)^{2}-2 \cdot \frac{1}{\lambda} \cdot \frac{P_{0}}{\lambda^{2}} \cdot u_{\lambda, P_{0}}} .
$$

The last term denotes the correlation terms with covariance $\mathrm{u}_{\lambda}, \mathrm{P}_{0}$, a term which gives a measure for the correlation of two variables. This problem was solved analytically after linearization of equation 2; therefore, the individual errors for $\mathrm{P}_{0}$ and $\lambda$ can be written as

$$
\begin{aligned}
& \sigma_{\lambda}=\sqrt{\left(n \cdot \frac{1}{n-2} \cdot \frac{\sum\left(C-\lambda t_{i}-Y_{i}\right)^{2}}{n \sum t_{i}^{2}-\left(\sum t_{i}\right)^{2}}\right)} \\
& \sigma_{P_{0}}=P_{0} \cdot \sigma_{C},
\end{aligned}
$$

where

$$
\begin{aligned}
& \sigma_{C}=\sqrt{\sum t_{i}^{2} \cdot \frac{1}{n-2} \cdot \frac{\sum\left(C-\lambda t_{i}-Y_{i}\right)^{2}}{n \sum t_{i}^{2}-\left(\sum t_{i}\right)^{2}}}, \\
& Y|t|-C-\lambda t \\
& \ln \mathrm{P}_{0}=\mathrm{C} .
\end{aligned}
$$

Under the assumption that the error of the dose measurement is negligible, $\sigma_{\mathrm{A}}$ (displayed as percent of the total area under the curve) also represents the error of the calculated GFR.

\section{GFR Measurements}

Three blood samples from 133 patients aged between 1 and 76 years examined at the Vienna General Hospital were collected 120, 180 and $240 \mathrm{~min}$ after the administration of ${ }^{51} \mathrm{Cr}$-EDTA. The three-sample GFR and the corresponding fit error, as well as the two-sample GFR from the blood samples collected at 180 and 240 min, were calculated using GFRcalc. The GFR was corrected for BSA according to the formula described by Mosteller [8]. In order to validate the GFR calculation process of the software, the calculation was performed twice, once with the previous routinely used method based on a handwritten slope-intercept method and once with GFRcalc. The fit error was compared to the Pearson's correlation coefficient $(\mathrm{r})$, which provides a measure of the linear correlation between two normally distributed values [in our case: times $t_{i}$ and the logarithm of the measured plasma concentrations $\mathrm{P}\left(\mathrm{t}_{\mathrm{i}}\right)$ ] and to the difference (mean \pm SD) between the GFR of the threesample and the two-sample method. For the single-sample GFR, several formulae [15-18] were used. The single-sample method is based on the principle that there exists an inverse relationship between GFR and plasma concentration at any fixed time point [15], where the variances in the area under the exponential curve are minimized when the chosen sampling time is roughly the biological half-life of the ligand divided by the natural logarithm of 2. In our study, the biological half-life calculated from the three-sample method was found to be $142 \pm 76 \mathrm{~min}$, resulting in a sampling time for the single-sample method of approximately $200 \mathrm{~min}$. Therefore, the sample collected 180 min after administration of ${ }^{51} \mathrm{Cr}$ EDTA was chosen for all single-sample calculations. 


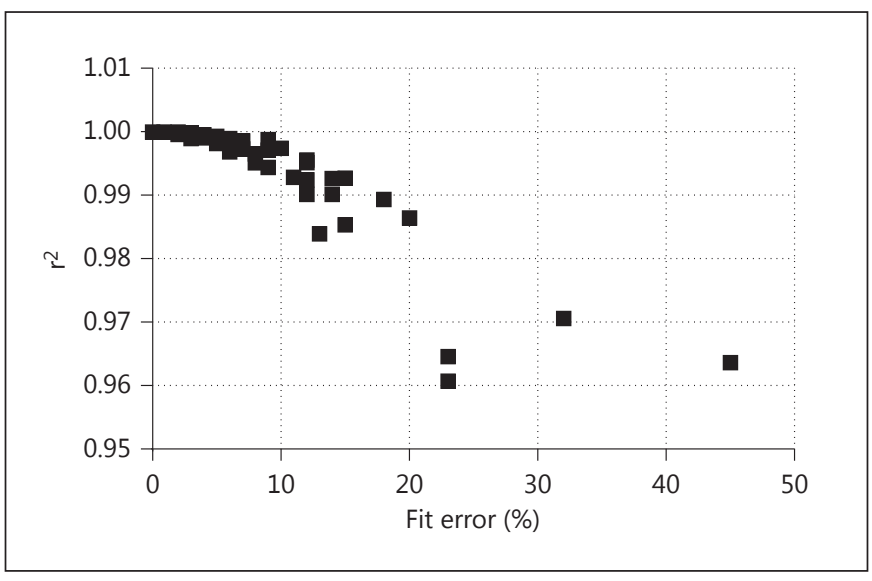

Fig. 2. Fit error from the GFRcalc calculation with three blood samples versus the correlation coefficient $r^{2}$. A value of $r \geq 0.985$ is considered as significantly correlated.

Table 1. Difference between the three-sample corrected GFR and the single-sample GFR calculated according to the formula of Watson [16]

\begin{tabular}{ll}
\hline $\begin{array}{l}\text { Three-sample GFR, } \\
\mathrm{ml} / \mathrm{min} / 1.73 \mathrm{~m}^{2}\end{array}$ & $\begin{array}{l}\text { Difference between the single- and the } \\
\text { three-sample method (means } \pm \mathrm{SD}), \\
\text { \% of the three-sample GFR }\end{array}$ \\
\hline $1-60$ & $25 \pm 27$ \\
$60-140$ & $14 \pm 13$ \\
$140-213$ & $17 \pm 13$ \\
$60-213$ & $15 \pm 14$ \\
$1-213$ & $17 \pm 17$ \\
\hline
\end{tabular}

The results are based on 133 patients. Note: a corrected GFR of $213 \mathrm{ml} / \mathrm{min} / 1.73 \mathrm{~m}^{2}$ was the highest GFR measured.

\section{Results}

No differences in GFR were found between the previous routinely used method and that calculated with GFRcalc. Of the 133 patients, 30 (23\%) had insufficient kidney function with a GFR $<60 \mathrm{ml} / \mathrm{min}$. The error of fit was as follows: extremely high in 1 patient: $62 \%$; in 6 patients between 20 and $45 \%$; in 13 patients between 10 and $20 \%$, and in the remaining 113 patients $<10 \%$. For the patient with insufficient kidney function and an error of fit of $62 \%$, it was not possible to find an error in the GFR measurement procedure, such as an incorrect counter reading or inaccurate time of sample collection. A repetition

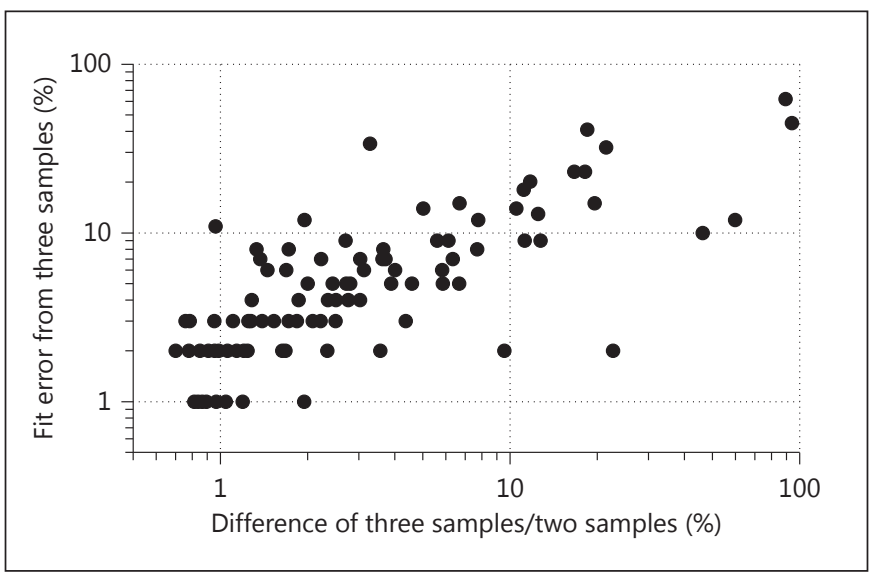

Fig. 3. The fit error from the three-sample method is plotted against the difference of the two-sample GFR to the three-sample GFR in percent of the three-sample result. The coefficient of determination $\left(\mathrm{r}^{2}\right)$ is $0.57(\mathrm{p}<0.0001)$.

of the measurement resulted in normal kidney function with an error $<10 \%$. The calculated biological half-life varied between 48 and $436 \mathrm{~min}$ in the 133 patients.

The error of the fit with three blood samples calculated with GFRcalc from 132 patients (except the patient with an error of $62 \%$ ) is plotted against the correlation coefficient $\mathrm{r}^{2}$ (fig. 2). For fit errors $<10 \%$, the correlation between the measured times $t_{i}$ and the measured blood sample activity $\mathrm{P}\left(\mathrm{t}_{\mathrm{i}}\right)$ was always significantly high with $\mathrm{r}^{2} \geq$ 0.985 . However, for high fit errors of $10-35 \%$, the correlation coefficient partially gave ambiguous results, e.g. a high value near 0.995 , while the fit error is either 10 or $18 \%$.

In case of the single-sample method, according to the Watson [16] formula, the results had the smallest deviations from the three-sample method with an averaged discordance of $17 \%$ (table 1). No relationship between the discordance of the single-sample GFR and the threesample GFR and the fit error from the three-sample method was found. Additionally, the comparison between the single-sample method taken $180 \mathrm{~min}$ after ${ }^{51} \mathrm{Cr}$-EDTA administration and that taken after $240 \mathrm{~min}$ did not show any significant difference (on average 6\%; $\mathrm{p}<0.001$ ).

The difference (mean \pm SD) between the GFR of the three-sample method and the GFR of the two-sample clearance was compared with the fit error of the threesample calculation. There was a positive relationship with greater discordance between the two-sample GFR measurement at higher fit errors for the three-sample GFR 
(fig. 3). In patients with a fit error $<10 \%$ for the threesample GFR, there was a difference of $2 \%$ between the GFRs resulting from two- and three-sample GFRs.

\section{Discussion}

Fit errors arise from random and systematic errors during the measurement procedure; the latter often cannot be eliminated as the daily working routine of technicians cannot be performed under controlled laboratory conditions. Due to the long-lasting counter measurement, the statistical (random) error is taken as negligible and the calculated error mainly represents the systematic error of the procedure.

There were discrepancies between the correlation coefficient and the fit error, in particular for fit errors $>10 \%$, probably because the correlation coefficient only evaluates the deviation of the mean value of a data set and ignores the gradient of the curve. The steeper the curve (at a constant offset), the greater the effect of variations in a data point. According to Fleming et al. [4], results with a correlation coefficient of $\mathrm{r}^{2}<0.985$ would be classified as not significantly correlated and should, therefore, be rejected, i.e. one would check the input data and the measurement routine. Because the gradient of the curve is taken into account by the fit error calculation, the latter is an advantage over determining the correlation coefficient. Regarding our results, we would suggest to check the data for fit errors $>10 \%$. The calculation of the fit error is conceptually more complicated than the calculation of the correlation coefficient; nevertheless, the former is more reliable in particular for ambiguous results.

The relatively high deviation (17\%) of the single-sample compared to the three-sample method supports the fact that the single-sample method is not recommended at low GFR values [4]. Also, a very weak relationship between the discordance between the single-sample and the three-sample GFR and the fit error from the three-sample method could be found $(r<0.5)$. This might be due to the fact that the single-sample method is prone to errors: the chosen sampling time of $180 \mathrm{~min}$ for its calculation is unlikely to be appropriate in all cases because the biological half-life differed between 48 and $436 \mathrm{~min}$ in the 133 patients, which would correspond to optimal individual sampling times of 70-632 min in order to minimize errors. However, it is not possible to predict whether a patient will have low or high renal function, therefore this sampling time was chosen for all patients. Thus, the sin- gle-sample method is not appropriate to assess the quality of two- or three-sample GFR measurements.

Finally, the slope-intercept method was applied to two blood samples collected at 180 and 240 min. Both methods delivered a similar result at fit errors $<10 \%$, but when the data had high fit errors, a difference in the results of the two methods was observed. This may be due to irregularities in the clinical routine, statistical outliers or deficits in the devices used. An advantage of automatically calculating the fit error is that it requires less effort than repeating the GFR calculation using data from a reduced number of samples.

The accuracy of the slope-intercept method with two or more blood samples is limited, because there are several models describing the decline in the plasma concentration [19]. However, the slope-intercept method applied to blood plasma samples collected at least $120 \mathrm{~min}$ after the administration of ${ }^{51} \mathrm{Cr}$-EDTA is suggested as the best compromise between accuracy and simplicity, and it is a repeatable and reliable method $[4,20,21]$ with reproducibility of GFR measurement $\leq 10 \%$. According to our data, we can conclude that a high fit error or data point deviating from the exponential curve is most likely related to an error in the measurement process, such as radioactive contamination close to the measurement devices, a wrong dilution factor, an uncalibrated or wrongly adjusted device or a typing error in the measurement protocol. It is important to note that a small error does not necessarily indicate an accurate measurement, because an incorrect measurement can randomly produce small fit errors. However, a high error $(>10 \%)$ indicates likely errors in the GFR measurement and calculation.

Additionally, a higher number of samples may positively influence the quality of the GFR results, because outlying data points would exert less influence on the results. A small number of samples in general suffers from a lack of reliability, even if the individual data points have a good counting statistic. After obtaining an unexpected high fit error, it is not always possible to immediately find out where in the GFR measurement procedure the error has occurred. Nevertheless, a data set of at least three blood samples is the only way to quantify irregularities in the process, and the fit error is a simple mathematical tool which helps to identify them. The collection of three blood samples to determine GFR is a compromise between maintaining patient comfort and ensuring accuracy and reliability. 


\section{Conclusion}

According to our results, the GFR measurement should be performed with at least three blood samples in order to identify possible errors in the measurement procedure. We conclude that the three-sample GFR with a fit error $<10 \%$ may be taken as reliable, and, at higher errors of fit, one should check the devices, the clinical procedure or other sources for systematic errors. Particularly in cas- es where the obtained GFR measurement is $<60 \mathrm{ml} / \mathrm{min}$, a valid assessment of the quality and reliability of the measurement is important as the GFR value may affect patient management. There are alternative methods to check GFR measurement for errors. However, GFRcalc offers the advantage that the error of the fit is automatically determined, which provides information about the quality of the GFR measurement and may facilitate classification of the diagnosis for the physician.

\section{References}

1 Garnett ES, Parsons V, Veall N: Measurement of glomerular filtration-rate in man using a ${ }^{51}$ Cr-edetic-acid complex. Lancet 1967;1:818819.

2 Brochner-Mortensen J, Giese J, Rossing N: Renal inulin clearance versus total plasma clearance of ${ }^{51} \mathrm{Cr}$-EDTA. Scand J Clin Lab Invest 1969;23:301-305.

-3 Anger G, Senf L, Schmidt U, et al: The clinical value of 51 chrome-EDTA clearance (in German). Z Gesamte Inn Med 1976;31:308-310.

4 Fleming JS, Zivanovic MA, Blake GM, et al: Guidelines for the measurement of glomerular filtration rate using plasma sampling. Nucl Med Commun 2004;25:759-769.

5 Brochner-Mortensen J: A simple method for the determination of glomerular filtration rate. Scand J Clin Lab Invest 1972;30:271274.

-6 Brochner-Mortensen J, Haahr J, Christoffersen J: A simple method for accurate assessment of the glomerular filtration rate in children. Scand J Clin Lab Invest 1974;33:140 143.

7 Jodal L, Brochner-Mortensen J: Reassessment of a classical single injection ${ }^{51} \mathrm{Cr}$-EDTA clearance method for determination of renal function in children and adults. Part I: analytically correct relationship between total and one-pool clearance. Scand J Clin Lab Invest 2009;69:305-313.

\footnotetext{
Mosteller RD: Simplified calculation of body surface area. N Engl J Med 1987;317:1098.

9 DuBois D, DuBois EF: A formula to estimate the approximate surface area if height and weight be known. Arch Intern Med 1916;17: 863-871.
}

$>10$ Haycock GB, Schwartz GJ, Wisotsky DH: Geometric method for measuring body surface area: a height-weight formula validated in infants, children and adults. J Pediatr 1978; 93:62-66.

11 Gehan EA, George SL: Estimation of human body surface area from height and weight Cancer Chemother Rep 1970;54:225-235.

12 Boyd E: The Growth of the Surface Area of the Human Body. Minneapolis, University of Minnesota Press, 1935.

13 Levey AS, Coresh J, Balk E, et al: National Kidney Foundation practice guidelines for chronic kidney disease: evaluation, classification, and stratification. Ann Intern Med 2003;139: 137-147.

14 Delanaye P, Schaeffner E, Ebert N, et al: Normal reference values for glomerular filtration rate: what do we really know? Nephrol Dial Transplant 2012;27:2664-2672.
15 Russell CD, Bischoff PG, Kontzen FN, et al: Measurement of glomerular filtration rate: single injection plasma clearance method without urine collection. J Nucl Med 1985;26: 1243-1247.

16 Watson WS: A simple method of estimating glomerular filtration rate. Eur J Nucl Med 1992;19:827.

17 Ham HR, Piepsz A: Estimation of glomerular filtration rate in infants and in children using a single-plasma method. J Nucl Med 1991;32: 1294-1297.

18 Hamilton D, Miola UJ: Body surface correction in single-sample methods of glomerular filtration rate estimation. Nucl Med Commun 1999;20:273-278.

19 Wanasundara SN, Wesolowski MJ, Puetter $\mathrm{RC}$, et al: The early plasma concentration of ${ }^{51} \mathrm{Cr}$-EDTA in patients with cirrhosis and ascites: a comparison of three models. Nucl Med Commun 2015;36:392-397.

20 Chantler C, Baratt TM: Estimation of glomerular filtration rate from plasma clearance of 51-chromium edetic acid. Arch Dis Child 1972;47:613-617.

21 Bird NJ, Peters C, Robert Michell A, et al: Comparison of GFR measurements assessed from single versus multiple samples. Am J Kidney Dis 2009;54:278-288. 